\title{
TRAVIESAS MONOBLOQUE POLIVALENTE: ESTUDIO TEÓRICO Y EXPERIMENTAL EN EL ICCET/CSIC. ESPAÑA
}

\author{
(MONOBLOCK RAILWAY SLEEPERS FOR CHANGEABLE TRACK WIDTH)
}

Fecha de recepción: 18-111-91

\begin{abstract}
RESUMEN
En este artículo se describen los trabajos realizados en el ICCET por encargo de RENFE y con objeto de definir y caracterizar una traviesa monobloque polivalente prefabricada de hormigón.

El trabajo consta de dos partes bien diferenciadas:

- La primera es el estudio teórico que nos conduce al dimensionamiento de las distintas secciones de la traviesa.

- En la segunda parte se hace un estudio experimental con vistas a la comprobación del dimensionamiento efectuado, asi como de las bases de cálculo utilizadas.
\end{abstract}

\section{SUMMARY}

The works ordered by RENFE, and carried out at the ICCET, are described here. These works are for defining and caracterizing a presasted all-purpose monoblock sleeper.

The work has two parts well different:

- The first one is the theoretical study that leads us to the sizing of the different sections of the sleeper.

- In the second one an experimental study is done in order to check the sizing as well as the basis used in the calculation.

\section{INTRODUCCION}

EI ICCET ha realizado recientemente, para RENFE, un estudio teórico y experimental con objeto de definir una traviesa monobloque polivalente de hormigón pretensado, que sirvió de base de partida para adoptar decisiones técnicas sobre el cambio de ancho de vía en la red nacional. Esta traviesa se concibió de manera que pudiera soportar, indistintamente, tanto el tráfico en vía de ancho internacional $(1.435 \mathrm{~mm})$, como el tráfico en vía de ancho RENFE $(1.668 \mathrm{~mm})$.

El estudio teórico consta, fundamentalmente, de dos partes. Una primera dedicada al análisis de la determinación de acciones y esfuerzos; y una segunda, dedicada a la elección del método de cálculo, su ejecución y la caracterización de la traviesa polivalente, junto con la definición de sus correspondientes prescripciones técnicas de fabricación.

El estudio experimental se planteó inicialmente con el propósito de verificar que los supuestos de partida para el cálculo de las traviesas eran los adecuados, y que los resultados de esos cálculos y el dimensionamiento realizado a partir de ellos eran suficientes para garantizar el buen comportamiento del elemento estructural durante su vida útil.
La realización de los ensayos se divide en tres fases. La primera corresponde a los ensayos estáticos y de fatiga sobre traviesas aisladas, con objeto de comprobar experimentalmente el comportamiento mecánico de las mismas y su correspondencia con los resultados obtenidos en el análisis teórico. La segunda corresponde a los ensayos estáticos y dinámicos sobre un tramo de vía montado sobre balasto en laboratorio. Por último, la tercera, recogerá los ensayos programados en un tramo real de vía.

En este trabajo (que sirvió de base para la presentación de dos ponencias al Simposio Internacional de traviesas ferroviarias prefabricadas de hormigón, celebrado en madrid del 8 al 11 de abril de 1991) se describe el estudio teórico de la traviesa polivalente, las bases y métodos de cálculo utilizados, y la determinación de esfuerzos y dimensionamiento en las distintas secciones de la traviesa. Además se describen los ensayos realizados, y la instrumentación y equipos utilizados en la materialización de los mismos.

Quedan pendientes de completar los ensayos sobre el tramo de vía en laboratorio y los ensayos en el tramo real de vía, que serán objeto de un trabajo posterior. 


\title{
TRAVIESAS FERROVIARIAS PREFABRICADAS DE HORMIGÓN
}

\author{
José Pedro Gutiérrez Jiménez, José Quereda Laviña, Francisco Morán Cabré, \\ Cecilio López Hombrados, Carlos Diaz Paniagua y Daniel Ribé Benages, \\ Ingenieros de Caminos \\ ICCET/CSIC
}

\section{ANTECEDENTES}

En el otoño de 1988, el Ministerio de Transporte, Turismo y Comunicaciones consideró la posibilidad de cambiar el ancho de la vía de la Red Nacional de los Ferrocarriles Españoles, que en la actualidad es de 1,668 m, a una separación idéntica a la de los ferrocarriles de Europa Occidental, cuya medida es 1,435 metros.

Como consecuencia de esta decisión, RENFE se puso en contacto con el Instituto de Ciencias de la Construcción Eduardo Torroja, ICCET, para analizar la viabilidad técnica de la operación, desde los puntos de vista mecánico y operativo. En un primer momento el Departamento de Investigación y Desarrollo de la Dirección de Ingeniería Civil de RENFE analizó diferentes soluciones eligiendo una de ellas, la diseñada por su Ingeniero José Julián Mendoza, solución que desde ese momento es conocida como "traviesa JJM".

A partir de esta elección, la Dirección de Ingeniería Civil de RENFE y el Departamento de Análisis Experimental de Estructuras del ICCET, acuerdan un plan de actuación que se concreta en desarrollar un Proyecto de Investigación dividido en dos Contratos de Investigación: el primero de carácter teórico y el segundo de carácter aplicado. Este plan se pone en marcha en diciembre de 1988 y continúa en la actualidad con el desarrollo de las últimas fases de ensayos en vía.

El modelo de traviesa polivalente, sobre la que se realizaron los primeros estudios, fue la denominada JJM.

La primera fase del proyecto de investigación se encuadra dentro del estudio y desarrollo teórico de los elementos estructurales de hormigón, con el objeto final de la definición de una traviesa monobloque de hormigón, con carácter polivalente para los anchos de vía RENFE e INTERNACIONAL, capaz de soportar los esfuerzos estáticos y dinámicos que corresponden al paso de los trenes que circulan actualmente por nuestro país a las velocidades habituales y hasta una velocidad máxima de $180 \mathrm{~km} / \mathrm{h}$ en ancho RENFE y de las que podrán obtenerse en el futuro de hasta $300 \mathrm{~km} / \mathrm{h}$ en ancho INTERNACIONAL.

Para la realización de los cálculos de verificación del primer modelo, la traviesa JJM propuesta por RENFE, al ICCET le fueron aportados los planos geométricos y las características básicas correspondientes definidas, en el momento de iniciación del Proyecto.

Después de varias reuniones entre técnicos de ambos Organismos, RENFE e ICCET, se decidió que el procedimiento de cálculo sería uno de los métodos simplificados que, sancionados por la experiencia, estuviera en uso en alguna de las administraciones ferroviarias de nuestro entorno, y que se eligiría por los investigadores responsables del Proyecto en función de la bondad contrastada del método y de la disponibilidad del equipamiento necesario para su desarrollo.

A partir de los estudios y los avances de resultados obtenidos se redefinieron las características geométricas y mecánicas de la sección de la traviesa en los dos supuestos, de armadura pretesa y de armadura postesa, llegando hasta definir las especificaciones técnicas básicas para la fabricación de los prototipos necesarios para posibilitar la realización de la fase experimental del proyecto, cuyo alcance y contenido se definieron también en este estudio de la investigación.

Por último debe indicarse que los resultados de los ensayos programados, que iban a realizarse a continuación, permitirian confirmar o no la validez de las hipótesis realizadas en este estudio, y en su caso proporcionarian información para decidir sobre la idoneidad del dimensionamiento propuesto.

Este trabajo ha sido realizado por los equipos de investigación "Análisis experimental de estructuras" y "Vida residual de estructuras afectadas por problemas 
patológicos" del proyecto P-880029, financiado por la DGICYT, que se desarrolla en el Instituto Eduardo Torroja del CSIC.

\section{BASES DE CÁLCULO}

Para el cálculo de proyecto de una traviesa es lógico aplicar modelos y métodos rigurosos. A estos efectos puede usarse un programa basado en el MEF tridimensional, considerando las acciones producidas por los ejes reales sobre los railes, que a su vez descansan en las traviesas, las cuales se apoyan sobre el balasto y éste sobre el terreno constituido por varias capas. El análisis debe ser no lineal (teniendo en cuenta ecuaciones constitutivas adecuadas para los distintos materiales), dinámico (considerando debidamente las características del material móvil y el efecto del estado de conservación de la via) y probabilista. Especial atención debe prestarse a los efectos diferidos en el tiempo (asientos del terreno, fluencia del hormigón, relajación y fatiga del acero).

En nuestro caso, se trata de predimensionar una traviesa prototipo y someterla a un programa de ensayos para analizar su comportamiento. Con los resultados se realizará luego el proyecto de la traviesa definitiva sobre bases rigurosas. Por tanto, se ha realizado un cálculo práctico simplificado. La traviesa se ha asimilado a un elemento lineal (viga) sobre base elástica. Dicho elemento se ha sometido a un análisis lineal elástico de primer orden (se trata de una pieza de hormigón pretensado que en servicio se mantiene con tensiones alejadas de las de rotura). El cálculo se realiza sobre bases deterministas, aplicando los coeficientes de seguridad parciales habituales.

La normativa aplicada es la vigente en España: Instrucciones EH-88 y EP-80 (versión modificada de 1986).

Se ha considerado un hormigón prefabricado para pretensado, de alta resistencia inicial. Su resistencia caracteristica a compresión se ha tomado igual a los 600 $\mathrm{kp} / \mathrm{cm}^{2}$ en probeta cilindrica normalizada. Su resistencia de cálculo a tracción se ha tomado igual a 30 $\mathrm{kp} / \mathrm{cm}^{2}$, valor muy del lado de la seguridad para tener en cuenta el efecto desfavorable de la fatiga. Como diagrama tensión-deformación se ha tomado el ParábolaRéctángulo a efectos de las comprobaciones en el estado límite último.

En cuanto al acero, para armaduras pretesas, que serán alambres de $6,9 \mathrm{~mm}$ de diámetro, se ha considerado una carga de rotura de $170 \mathrm{kp} / \mathrm{mm}^{2}$. Para armaduras postesas, que serán barras de $9,7 \mathrm{~mm}$ de diámetro, se ha considerado una carga de rotura de $150 \mathrm{kp} / \mathrm{mm}^{2}$. Como diagramas tensión-deformación de estos aceros se han usado los definidos por puntos a partir de ensayos de tracción sobre el material real.
Para las comprobaciones frente al estado límite último se ha tomado un coeficiente de mayoración de acciones $\gamma_{f}=1,6$; un coeficiente de minoración de resistencias del hormigón $\gamma_{c} 1,5$ (podría haberse bajado a 1,4 por tratarse de elementos prefabricados en instalaciones permanentes con control intenso); y un coeficiente de minoración de resistencias del acero $\gamma_{\mathrm{s}}=$ $=1,1$ (control a nivel intenso). Para las comprobaciones frente a los estados límites de servicio se han tomado coeficientes de seguridad iguales a la unidad.

En cuanto a las acciones consideradas, las especificaciones de anteproyecto de RENFE establecian tres comprobaciones distintas. Por una parte, vía de ancho RENFE y velocidad de proyecto de $180 \mathrm{~km} / \mathrm{h}$, con una carga estática por eje de 22,5 t. Por otra, vía de ancho internacional y velocidad de proyecto de $300 \mathrm{~km} / \mathrm{h}$, con una carga estática por eje de 20 t y una carga dinámica de 17,85 t por rueda. Por otra, vía de ancho internacional y velocidad de proyecto de $200 \mathrm{~km} / \mathrm{h}$, con una carga estática por eje de 22,5 t y una carga dinámica de $17,85 \mathrm{t}$.

Se ha supuesto que la carga estática media sobre rueda $Q_{\text {med }}$ es la mitad de la carga estática por eje aumentada en un 20 por 100 por efectos de incremento de carga en curva. La carga máxima sobre rueda $Q_{\max }$ se obtiene de la media aplicando la expresion:

$$
Q_{\max }=Q_{\text {med }}(1+\text { t.s. } \varnothing)
$$

siendo:

$s=$ coef. que depende del estado de la vía. Para vía en buen estado puede tomarse $s=0,10$; para vía en mal estado, $s=0,30$; para estado intermedio, $s=0,15$.

$\mathrm{t}=$ coef. de seguridad estadística. Para un nivel de confianza del $95 \%$ puede tomarse $t=0,2$; para un nivel de confianza del $99,7 \%$ puede tomarse $t=0,3$.

$\varnothing=$ factor de velocidad, que es función de la velocidad de proyecto.

La aplicación de estas tres situaciones de proyecto ha conducido a considerar, para ancho de vía RENFE, unas cargas medias sobre carriles de $13,5 \mathrm{t}$ y unas cargas máximas sobre carriles de 24,8 t. Para ancho de vía internacional, unas cargas máximas sobre carriles de 26,7 toneladas y unas cargas dinámicas de 17,85 toneladas.

La carga sobre la traviesa se obtiene considerando que el carril es una viga flotante sobre lecho elástico, mediante la expresión:

siendo:

$$
P=a \cdot Q /(2 L)
$$

$a=60 \mathrm{~cm}=$ distancia entre traviesas;

$L=(4 \mathrm{El} /(\mathrm{b} . \mathrm{c})) \cdot(1 / 4)=$ longitud virtual de la viga flotante;

$E=21.000 \mathrm{kN} / \mathrm{cm}^{2}=$ módulo de elasticidad del carril; 
I = inercia del carril. Para carril UIC-60, I = $3055 \mathrm{~cm}^{4}$; para carril UIC-54, I = $2364 \mathrm{~cm}^{4}$;

$\mathrm{b}=$ ancho equivalente de la traviesa;

$c=$ coeficiente de balasto del suelo.

Para suelo bueno, $C=100 \mathrm{~N} / \mathrm{cm}^{3}$; para suelo rígido, $\mathrm{C}=300 \mathrm{~N} / \mathrm{cm}^{3}$;

La aplicación de esta fórmula conduce a una carga sobre traviesa $P$ igual a $0,515 \mathrm{Q}$, siendo $\mathrm{Q}$ la correspondiente carga sobre carril. Sin embargo, para quedar del lado de la seguridad, la carga de cálculo sobre traviesa se toma en todo caso no menor de la carga media sobre carril $Q_{\text {med }}=13,50$ t para vía de ancho RENFE, y de la carga dinámica sobre carril $Q_{\text {din }}=17,85 \mathrm{t}$ para vía de ancho internacional.

\section{DETERMINACION DE ESFUERZOS. DIMENSIONAMIENTO Y COMPROBACION}

Este estudio se ha realizado para los dos anchos de vía considerados: ancho RENFE y ancho INTERNACIONAL. En cada ancho se han utilizado dos tipos de carril, UIC 60 y 54 , dos hipótesis de subsuelo que corresponden respectivamente a un subsuelo bueno y a un subsuelo rígido, y dos hipótesis de distribución de presiones bajo la traviesa para determinar las solicitudes en la misma.

\section{Longitud de reparto de la carga bajo carril}

La carga puntual actuando sobre el carril se reparte $o$ distribuye en una longitud que depende de la superficie de apoyo del carril, de la geometría de la placa intermedia de apoyo, y de las dimensiones de la traviesa.
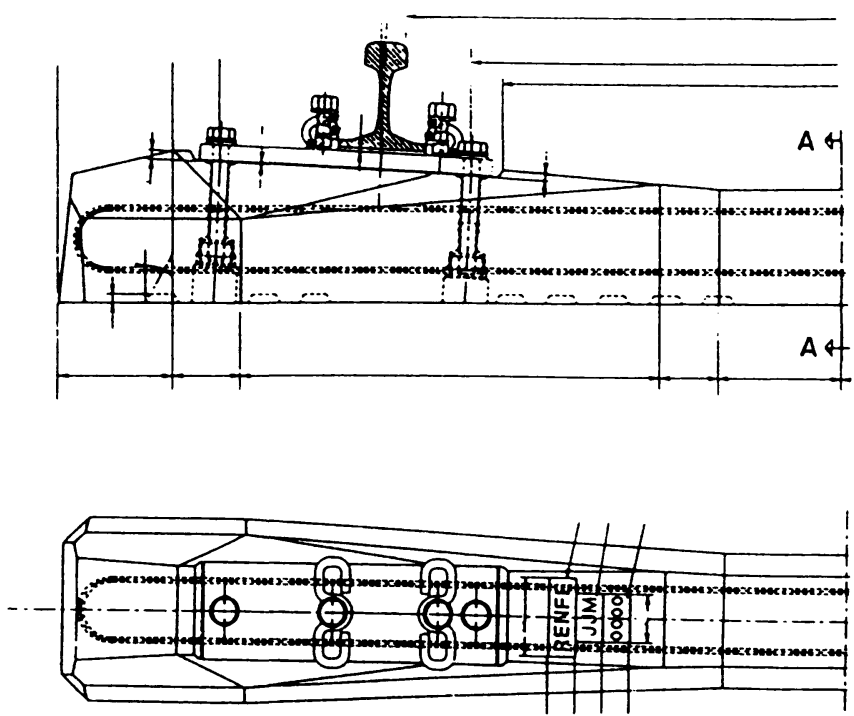

Fig. 1
Normalmente se consideran 2 hipótesis:

- Un reparto a $45^{\circ}$ hasta cortar el eje de centros de gravedad de la traviesa.

- Un reparto a $60^{\circ}$ que se prolonga hasta el fondo de la traviesa.

Para una de las traviesas analizadas tipo JJM (Fig. 1), y limitando el apoyo bajo carril a $150 \mathrm{~mm}$, las longitudes de reparto que se obtienen, en la doble hipótesis de utilización de la traviesa con ancho RENFE y con ancho INTERNACIONAL, son:

REPARTO
$60^{\circ}$
$45^{\circ}$

\section{ANCHO RENFE \\ $\mathrm{Dr}=446,75 \mathrm{~mm}$ \\ $\mathrm{Dr}=440,29 \mathrm{~mm}$}

\section{ANCHO INTERNACIONAL \\ $\mathrm{Dr}=460,04 \mathrm{~mm}$ \\ $\mathrm{Dr}=452,48 \mathrm{~mm}$}

Para todos los casos posibles de estudio es conveniente cubrir las situaciones más desfavorables que puedan presentarse, lo que se consigue adoptando una longitud de reparto, Dr, no mayor de $440 \mathrm{~mm}$, que producirá un menor redondeo de la ley de momentos y en consecuencia mayores momentos en las secciones bajo carril.

\section{Determinación de momentos en la traviesa}

La distribución de presiones bajo traviesa es difícil de conocer. A efectos prácticos suelen considerarse distribuciones uniformes, suficientemente contrastadas, que depende entre otros factores del estado en que se encuentra el balasto.

Dos hipótesis son las utilizadas normalmente para determinar las solicitaciones pésimas en la traviesa (Fig. 2):

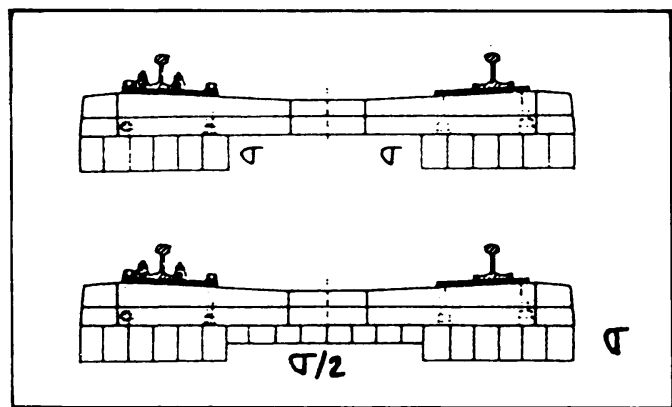

Fig. 2 
Hipótesis A:

La traviesa está sin apoyo en la zona central, y con una distribución de presiones uniforme en los extremos.

Hipótesis B:

La traviesa está totalmente en contacto con el balasto, con una distribución de presiones uniforme en los extremos, y una distribución de presiones uniforme en la zona central, de valor mitad a la existente en los extremos.

Establecidas las hipótesis anteriores, y para realizar un estudio sistemático de la traviesa tipo JJM, se desarrolló un programa de computador escrito en HP-BASIC para el ordenador HP-9816, que permite obtener las presiones, esfuerzos y tensiones en las secciones de la traviesa, en intervalos de $10 \mathrm{~cm}$ de longitud.

En general, la geometria de las traviesas de hormigón es complicada y conviene definir la geometría en planta de una forma simplificada a base de trapecios que permita de forma rápida tantear soluciones con distintas dimensiones.

La presión se determina estableciendo el equilibrio de fuerzas en la traviesa. Conocida la presión, la determinación de esfuerzos es inmediata.

Para la traviesa JJM, se alcanzan las siguientes presiones máximas bajo traviesa:

\section{HIPOTESIS ANCHO RENFE ANCHO INTERNACIONAL A $\quad 5,66 \mathrm{kp} / \mathrm{cm}^{2}$ $6,09 \mathrm{kp} / \mathrm{cm}^{2}$ \\ B $\quad 4,65 \mathrm{kp} / \mathrm{cm}^{2}$ \\ $5,62 \mathrm{kp} / \mathrm{cm}^{2}$}

MOMENTOS ANCHO DE VIA $1.668 \mathrm{~m}$

\begin{tabular}{|c|c|c|}
\hline $\mathbf{X}(\mathbf{m})$ & HIPOTESIS A & HIPOTESIS B \\
\hline $\mathbf{M a}(\mathbf{m} \cdot \mathbf{t})$ & Mb $\mathbf{( m \cdot t )}$ & \\
0,00 & 0,00 & 0,00 \\
, 10 &, 08 &, 07 \\
, 20 &, 34 &, 28 \\
, 30 &, 62 &, 48 \\
, 40 &, 76 &, 51 \\
, 42 &, 77 &, 50 \\
, 50 &, 75 &, 37 \\
, 60 &, 60 &, 06 \\
, 70 &, 35 &,- 39 \\
, 80 &, 21 &,- 74 \\
, 90 &, 20 &,- 98 \\
1,00 &, 20 & $-1,16$ \\
1,10 &, 20 & $-1,29$ \\
1,20 &, 20 & $-1,37$ \\
1,30 &, 20 & $-1,39$ \\
\hline
\end{tabular}

(C) Consejo Superior de Investigaciones Científicas Licencia Creative Commons 3.0 España (by-nc)
MOMENTOS ANCHO DE VIA $1.435 \mathrm{~m}$

\begin{tabular}{|c|c|c|}
\hline $\mathbf{X}(\mathbf{m})$ & HIPOTESIS $\mathbf{A}$ & HIPOTESIS B \\
\hline & Ma (m·t) & Mb $\mathbf{( m \cdot t )}$ \\
0,00 & 0,00 & 0,00 \\
, 10 &, 09 &, 08 \\
, 20 &, 37 &, 34 \\
, 30 &, 82 &, 76 \\
, 40 & 1,33 & 1,21 \\
, 50 & 1,66 & 1,43 \\
, 54 & 1,65 & 1,45 \\
, 60 & 1,65 & 1,40 \\
, 70 & 1,46 & 1,12 \\
, 80 & 1,05 &, 61 \\
, 90 &, 73 &, 17 \\
1,00 &, 55 &,- 13 \\
1,10 &, 51 &,- 30 \\
1,20 &, 51 &,- 40 \\
1,30 &, 51 &,- 43 \\
\hline
\end{tabular}
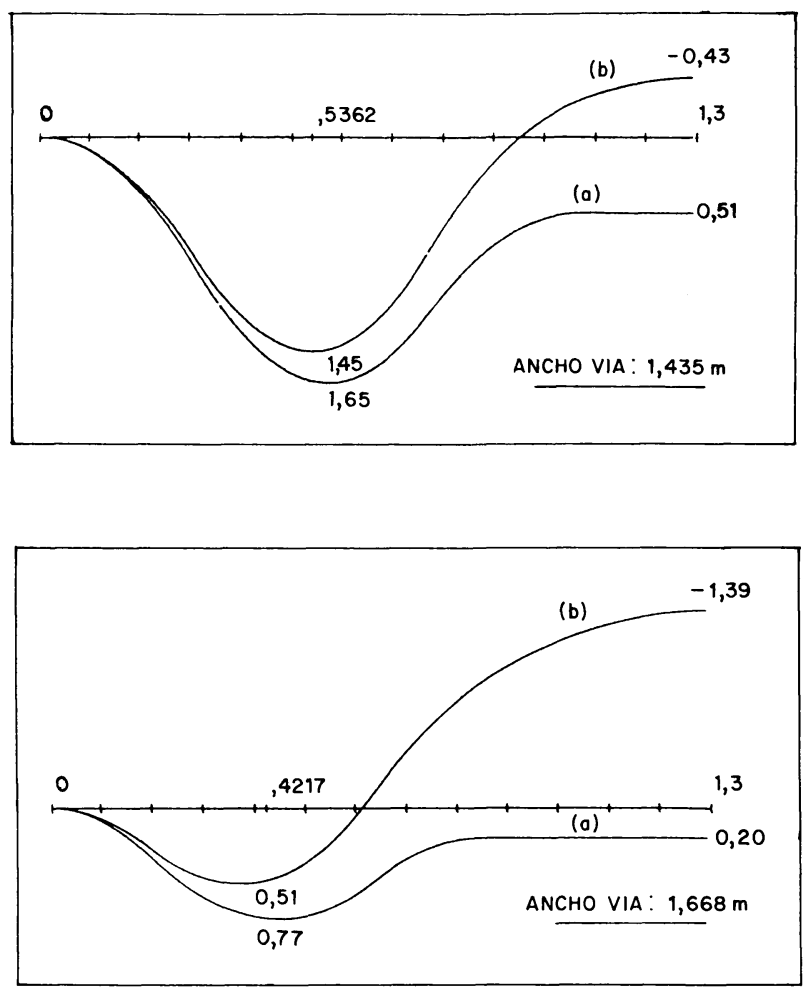

Fig. 3

MOMENTOS EN TRAVIESA (m.t)

\begin{tabular}{|c|c|c|c|c|}
\hline \multirow{2}{*}{ HIPOTESIS } & \multicolumn{2}{|c|}{ SECCION BAJO CARRIL } & \multicolumn{2}{c|}{ SECCION CENTRAL } \\
\hline \multirow{3}{*}{ A } & RENFE & INTERNAC. & \multicolumn{1}{c|}{ RENFE } & INTERNAC. \\
\cline { 2 - 5 } B & 0,77 & 1,65 & 0,20 & 0,51 \\
& 0,51 & 1,45 & $-1,39$ & $-0,43$ \\
\hline
\end{tabular}




\section{Determinación del pretensado en la traviesa}

La solución técnica del pretensado de las traviesas puede realizarse con armaduras pretesas o con armaduras postesas. Para la optimización de la posición pretensado es necesario proceder por tanteos. Para los casos estudiados se han elegido tres posiciones del centro de gravedad del pretensado, que referidos a la sección central de la traviesa tipo JJM, se sitúan a una altura de $95 \mathrm{~mm}, 100 \mathrm{~mm}$ y $105 \mathrm{~mm}$.

Para armaduras pretesas con 8 cables de pretensado de $6,9 \mathrm{~mm}$ de diámetro y $37,39 \mathrm{~mm}^{2}$ de sección se obtienen los siguientes resultados, para las tracciones máximas:

TRACCIONES MAXIMAS (kp/cm²)

\begin{tabular}{|c|c|c|}
\hline $\begin{array}{c}\text { ALTURA DE } \\
\text { PRETENSADO (mm) }\end{array}$ & $\begin{array}{c}\text { ANCHO } \\
\text { RENFE }\end{array}$ & $\begin{array}{c}\text { ANCHO } \\
\text { INTERNACIONAL }\end{array}$ \\
\hline 95 & $-39,37$ & $-20,52$ \\
100 & $-26,29$ & $-28,50$ \\
105 & $-13,77$ & $-36,58$ \\
\hline
\end{tabular}

Para armaduras postesas con 4 barras de $9,7 \mathrm{~mm}$ de diámetro se obtienen los siguientes resultados:

TRACCIONES MAXIMAS (kp/cm²)

\begin{tabular}{|c|c|c|}
\hline $\begin{array}{c}\text { ALTURA DE } \\
\text { PRETENSADO (mm) }\end{array}$ & $\begin{array}{c}\text { ANCHO } \\
\text { RENFE }\end{array}$ & $\begin{array}{c}\text { ANCHO } \\
\text { INTERNACIONAL }\end{array}$ \\
\hline 95 & $-48,50$ & $-27,77$ \\
100 & $-36,53$ & $-34,89$ \\
105 & $-24,96$ & $-42,11$ \\
\hline
\end{tabular}

La compatibilización de uso con ancho RENFE y con ancho INTERNACIONAL Ileva a considerar situaciones en donde las tensiones de tracción sean las mínimas posibles.

Para una altura del centro de gravedad del pretensado de $100 \mathrm{~mm}$ (Fig. 4) se obtienen las siguientes tensiones máximas de compresión y de tracción:

ARMADURAS PRETESAS

\begin{tabular}{|l|r|r|r|r|}
\hline \multirow{2}{*}{} & \multicolumn{2}{|c|}{ SECCION BAJO CARRIL } & \multicolumn{2}{c|}{ SECCION CENTRAL } \\
\cline { 2 - 5 } & \multicolumn{1}{|c|}{ RENFE } & INTERNAC. & \multicolumn{1}{c|}{ RENFE } & INTERNAC. \\
\hline TRACCION & 21,77 & $-28,50$ & $-26,29$ & 8,66 \\
COMPRESION & 105,44 & 170,73 & 182,46 & 179,23 \\
\hline
\end{tabular}

(c) Consejo Superior de Investigaciones Científicas Licencia Creative Commons 3.0 España (by-nc)

\section{ARMADURAS POSTERAS}

\begin{tabular}{|l|c|r|r|r|}
\hline \multirow{4}{*}{ TRACCION } & \multicolumn{2}{|c|}{ SECCION BAJO CARRIL } & \multicolumn{2}{c|}{ SECCION CENTRAL } \\
\cline { 2 - 5 } COMPRESION & RENFE & INTERNAC. & \multicolumn{1}{c|}{ RENFE } & INTERNAC. \\
\cline { 2 - 5 } & 15,32 & $-34,89$ & $-36,53$ & 3,66 \\
& 98,85 & 163,44 & 176,38 & 166,79 \\
\hline
\end{tabular}

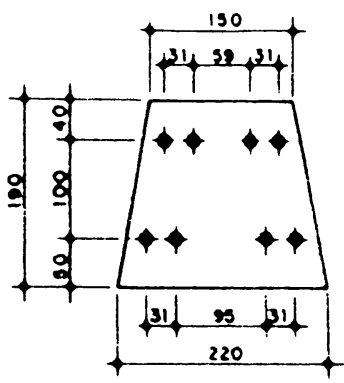

SECCION CENTRAL

SOLUCION PRETESADAS 8 cables $\varnothing 6,9$ Área $=37,39 \mathrm{~mm}^{2} /$ cable

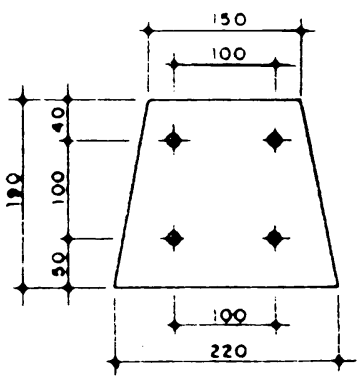

SECCION CENTRAL

SOLUCION POSTESADAS $4 \varnothing$ de $9,7 \mathrm{~mm}$ Área $=73,89 \mathrm{~mm}^{2} /$ cable
Fig. 4

\section{Momento último de rotura}

El coeficiente de seguridad a rotura de las secciones se calcula como cociente entre el momento último resistente de cada sección y el momento característico solicitante.

Se considera una resistencia característica del hormigón, $f_{\mathrm{ck}}$ de $500 \mathrm{kp} / \mathrm{cm}^{2}$ en probeta cilíndrica, $y$ una deformación última del hormigón, $\epsilon_{\mathrm{cu}}$, de $3 \%$.

Los coeficientes obtenidos, para las secciones bajo carril, y sección central, son:

\begin{tabular}{|c|c|c|}
\multicolumn{1}{c}{} & $\begin{array}{c}\text { ARMADURAS } \\
\text { PRETESAS }\end{array}$ & $\begin{array}{c}\text { ARMADURAS } \\
\text { POSTESAS }\end{array}$ \\
\hline SECCION & Mu/M & Mu/M \\
\hline BAJO CARRIL RENFE & 4,30 & 4,25 \\
BAJO CARRIL INTER. & 1,89 & 1,87 \\
CENTRAL MOM. + & 4,55 & 4,51 \\
CENTRAL MOM. - & 1,89 & 1,88 \\
\hline
\end{tabular}

\section{CONCLUSIONES}

Un estudio riguroso de las traviesas monobloque polivalente de hormigón pretensado requiere métodos avanzados de cálculo basados en el MEF tridimensional, que incorporen el análisis no lineal, dinámico y pro- 
babilista. No obstante, la combinación de métodos aproximados, suficientemente contrastados, y de un programa de ensayos para analizar el comportamiento de las traviesas, puede considerarse el procedimiento más adecuado para el dimensionamiento de prototipos, antes de adoptar diseños finales definitivos. Los métodos propuestos permiten, de forma rápida, estudiar soluciones que fijen las dimensiones óptimas de las traviesas.

Las soluciones propuestas para las traviesas pretensadas se encuentran bajo condiciones de servicio sometidas a unas tracciones máximas que pueden considerarse dentro del rango admisible, si bien en algunos casos, en sección bajo carril internacional $(34,89$ $\mathrm{kp} / \mathrm{cm}^{2}$ ) y en sección central con ancho de vía RENFE $\left(36,53 \mathrm{kp} / \mathrm{cm}^{2}\right)$, se superan la tensión de tracción admisible de $\left(30 \mathrm{kp} / \mathrm{cm}^{2}\right)$.

Sin embargo, debe tenerse en cuenta que para ancho internacional se ha considerado una carga dinámica de 17,85 t/rueda, sin realizar ningún reparto longitudinal entre traviesas y que para el ancho de vía RENFE se ha tomado la carga estática media de 13,50 t/rueda, en vez de la carga que se obtendría con un reparto entre traviesas, de valor 12,76 t/rueda.

Estas consideraciones hacen que las tensiones de tracción reales sean menores que las indicadas anteriormente, y por lo tanto se dé por válida la solución adoptada.

Por último, debe indicarse que los resultados de los ensayos programados confirmarán o no la validez de las hipótesis realizadas en este estudio, y en su caso proporcionarán información para decidir sobre la idoneidad del dimensionamiento propuesto.

\section{BIBLIOGRAFIA}

1.-ORE. Sollicitations des traverses en beton. Rapport n. 9. UTRECHT - 1969.

2.-B. PARZEFALL. Die Spannbetonschwellen B 55 y B70. Heft 51. Herausgegoben van O. Prof. - Dr. Ing. J. Eisenmann. Techniche Universität München - 1986.

3.-E. ALONSO, A. LÓPEZ PITA. El comportamiento aleatorio del balasto y su incidencia en el deterioro de una vía férrea que discurre sobre base rígida. E.T.S.I.C.C.P. Universidad Politécnica de Barcelona 1980.

4.-R. SAUVAGE. Calcul des traverses. ORE - 1968.

5.-K. GYLLTOFT. Working Group on Concrete Railway Sleepers. FIP. state of art report - 1987.

6.-FUNDACIÓN DE LOS FERROCARRILES ESPAÑOLES. Experiencias europeas en construcción y conservación de vías de alta velocidad y de tráfico pesado . 1989.

7.-J. I. RODRÍGUEZ MIRA. Traviesas de hormigón pretensado para ferrocarril. Hormigón y Acero n. 146. $1^{\text {er }}$ Trimestre 1983.

8.-F. OLIVEROS. Tratados de ferrocarriles. Editorial Rueda - 1980. 


\title{
TRAVIESAS MONOBLOQUE POLIVALENTES: ESTUDIO EXPERIMENTAL EN LABORATORIO
}

\author{
José Quereda Laviña, Cecilio López Hombrados, Carlos Díaz Paniagua, \\ Daniel Ribé Benages y José Pedro Gutiérrez Jiménez, Ingeniero de Caminos \\ ICCET/CSIC
}

\section{INTRODUCCION}

Con objeto de analizar el comportamiento de las traviesas monobloque polivalentes de hormigón pretensado, diseñadas para permitir el cambio de ancho de vía en la Red Nacional de los Ferrocarriles Españoles, se elaboró un Plan de Ensayos con el doble propósito de conocer el funcionamiento de la traviesa como elemento estructural aislado y del conjunto de ellas como superestructura ferroviaria.

Para este fin se elaboró un Plan de Ensayos, el cual se viene llevando a cabo hasta estos momentos en los que se están realizando los correspondientes de determinación de esfuerzos y deformaciones en un tramo de vía con circulación real de unidades ferroviarias de diversa índole.

Si bien en un principio el Plan de Ensayos se centraba en un determinado modelo de traviesa polivalente, tanto con armadura postesa como con armadura pretesa, este último modelo no llegó a ensayarse y, además, a lo largo de los meses transcurridos se ha producido una evolución de los diferentes modelos presentados, 10 que ha dado lugar a que el Plan se haya ido adaptando a este proceso y en la actualidad se centra en los modelos con armadura postesa que se vienen fabricando de forma industrial y se están colocando en la Red principal.

Una vez realizados, con resultados positivos, los estudios teóricos sobre el comportamiento mecánico de la traviesa, se procedió a la definición de las especificaciones necesarias para la fabricación de los primeros prototipos sobre los que se realizaron los ensayos iniciales.

Simultáneamente se habia ido elaborando el Plan de Análisis Experimental, con el alcance y contenido que más adelante se señala, en el que se tuvieron en cuenta los efectos estáticos, dinámicos y de fatiga a los que, presumiblemente, iba a estar sometida la traviesa a lo largo de su vida útil.

En esencia los datos de partida se basaron en la verificación del comportamiento de la traviesa frente a los esfuerzos estáticos y dinámicos que corresponden al paso de los trenes que circulan por nuestro país a las velocidades actuales y hasta una velocidad máxima de $180 \mathrm{~km} / \mathrm{h}$ en ancho RENFE y de $300 \mathrm{~km} / \mathrm{h}$ en ancho INTERNACIONAL, para lo cual se realizaron estudios experimentales tanto en laboratorio como en vía, agrupados en cuatro apartados:

- Ensayos estáticos en laboratorio.

- Ensayos de fatiga en laboratorio.

- Ensayos dinámicos en un tramo experimental preparado en laboratorio.

- Ensayos al paso de diferentes trenes en vía.

\section{PLANTEAMIENTO DE LA INVESTIGACION EXPERIMENTAL}

Dado que el conjunto de los resultados de los ensayos que se iban a realizar permitirian confirmar o no la validez de las hipótesis realizadas en el estudio teórico previo y que también iban a proporcionar información para decidir sobre la idoneidad del dimensionamiento propuesto, la investigación experimental se elaboró con el objetivo de comprobar el buen comportamiento del elemento estructural durante su vida útil, por lo que los ensayos se realizaron con tres planteamientos diferentes:

- Un primer grupo correspondía a ensayos en laboratorio tanto estáticos como dinámicos y de fatiga, sobre traviesas del nuevo diseño aisladas, al objeto de comprobar experimentalmente el comportamiento mecánico de las mismas y su correspondencia con los resultados obtenidos en el análisis teó- 
rico. También en laboratorio se realizaría una prueba estática y dinámica sobre un tramo de vía de unos $10 \mathrm{~m}$ de longitud colocado sobre balasto.

- Un segundo grupo correspondía a ensayos de información en un tramo del trazado de vía actual, con la finalidad de verificar el reparto de las acciones al paso de los vehículos entre traviesas adyacentes, así como el efecto medio de sustentación del balasto.

- El tercer grupo se realizaría en un tramo real en el que se hubieran colocado las nuevas traviesas, estudiando el rendimiento en la operación del cambio de ancho para verificar la velocidad de ejecución del proceso. Este grupo de pruebas lo realizó directamente el personal técnico de la Dirección General de Mantenimiento de RENFE.

El planteamiento de los ensayos a realizar recoge dos aspectos diferentes:

- Verificación experimental de las hipótesis realizadas para el cálculo de la traviesa y comparación de los resultados obtenidos con los esperados del diseño.

- Comportamiento real de la estructura de sustentación del carril, es decir, del conjunto de traviesas afectadas por la acción de una carga, situadas sobre el balasto en condiciones de uso habitual.

\section{PLAN DE ENSAYOS}

\begin{tabular}{|c|c|c|c|c|c|}
\hline \multicolumn{6}{|c|}{ NÚMERO DE MUESTRAS POR TIPO DE ENSAYO } \\
\hline \multicolumn{3}{|c|}{ ESPECIFICACIÓN DEL ENSAYO } & \multicolumn{3}{|c|}{ EDAD TRA. Y LUGAR DE ENSAYO } \\
\hline ENSAYO & TIPO & $\begin{array}{l}\text { SENTIDO } \\
\text { DE M. O } \\
\text { NÚM. CICLOS }\end{array}$ & $\begin{array}{c}7 \text { días } \\
\text { FÁBRICA }\end{array}$ & $\begin{array}{l}14 \text { dias } \\
\text { FÁBRICA }\end{array}$ & $\begin{array}{l}28 \text { dias } \\
\text { FÁBRICA }\end{array}$ \\
\hline \multirow{3}{*}{$E$} & \multirow{2}{*}{ E.MCV. } & E.MCV.P & 4 & 4 & 4 \\
\hline & & E.MCV.N & 4 & 4 & 4 \\
\hline & E.MBC. & E.MBC.P & $4+4$ & $4+4$ & $4+4$ \\
\hline \multirow{8}{*}{$F$} & \multirow{4}{*}{ F.MCV. } & F.MCV.C & & & $2+2$ \\
\hline & & F.MCV.Q & & & $2+2$ \\
\hline & & F.MCV.M & & & $2+2$ \\
\hline & & F.MCV.D & & & 3 \\
\hline & \multirow{4}{*}{ F.MBC. } & F.MBC.C & & & $2+2$ \\
\hline & & F.MBC.Q & & & $2+2$ \\
\hline & & F.MBC.M & & & $2+2$ \\
\hline & & F.MBC.D & & & 3 \\
\hline \multirow{2}{*}{$\mathrm{s}$} & S.I. & & & & 4 \\
\hline & S.D. & & & & 2 \\
\hline$P$ & P.V. & \multicolumn{4}{|c|}{ Instrumentación y análisis de 12 traviesas } \\
\hline \multirow{2}{*}{$\mathrm{E}$} & B.E. & \multicolumn{4}{|c|}{$\begin{array}{l}\text { Tramo de } 10 \text { metros montado en laboratorio } \\
\text { para ensayos estáticos y dinámicos }\end{array}$} \\
\hline & B.D. & \multicolumn{4}{|c|}{ Tramo de $1 \mathrm{~km}$ con paso de trenes } \\
\hline
\end{tabular}

(C) Consejo Superior de Investigaciones Científicas Licencia Creative Commons 3.0 España (by-nc)
Para resolver dichos aspectos se elaboró el Plan que figura en el cuadro siguiente, idéntico para las traviesas con armadura postesa confeccionadas por los cuatro fabricantes españoles que tradicionalmente venían suministrando a RENFE, y cuya explicación figura en el cuadro anterior.

Había un primer grupo de ensayos estáticos que se realizaron a los 7 y a los 14 dias de edad de las traviesas, respectivamente, en el laboratorio de la factoría donde se fabricaron. El mismo tipo de ensayos se realizó cuando las traviesas tenían más de 28 dias de edad, en las instalaciones del Instituto Eduardo Torroja.

Este primer grupo recoge tres ensayos diferentes:

- Comportamiento de la traviesa bajo el momento flector positivo en la sección central de la misma (E.MCV.P.).

- Comportamiento de la traviesa bajo el momento flector negativo en la sección central de la misma (E.MCV.N.).

- Comportamiento de la traviesa bajo momento flector positivo en la sección en la que se sitúa el carril (E.MBC.P.), para la situación de Ancho RENFE y Ancho INTERNACIONAL.

Un segundo grupo de ensayos se refiere al comportamiento a fatiga de las traviesas, realizándose los ensayos comprobando la sección central (MCV) tanto para los momentos positivos (2 ensayos), como negativos (2 ensayos) y el comportamiento de la sección en la que apoya el carril, en los dos supuestos de ancho estudiados, con 2 ensayos para cada uno de ellos. En ambos casos se analizó la influencia y evolución de dicha fatiga mediante la comparación del comportamiento de la traviesa después de un determinado número de ciclos, ensayándola hasta rotura con la configuración de ensayos estáticos descrita anteriormente.

Aparece a continuación un grupo de ensayos que se han denominado singulares, divididos en dos tipos:

- 4 ensayos a impacto, con la traviesa apoyada de forma inclinada, para verificar el comportamiento frente a acciones laterales producidas por efectos repentinos (S.I.).

- 2 ensayos de desgaste del material para verificar el efecto abrasivo del balasto (S.D.).

Se indica a continuación un ensayo (P.V.) previo en vía, con objeto de verificar el comportamiento real de alguna de las hipótesis realizadas: reparto de la carga entre traviesas adyacentes, efecto del asiento de la tra- 
viesa,... y un grupo de ensayos divididos en dos conjuntos:

- Ensayos en laboratorio sobre un tramo de unos $10 \mathrm{~m}$ construido al efecto (B.E. y B.D.), en el que se realizarían ensayos estáticos y dinámicos para verificar el comportamiento de las traviesas simulando las acciones reales.

- Por último, se previó realizar la instrumentación en las traviesas de un tramo de la Red en el que se colocarian las nuevas traviesas y en el que los vehículos podían circular a diferentes velocidades y con distintas cargas por eje (B.V.).

Hay que señalar también el hecho de que, por indicación expresa de RENFE, no se ensayó el modelo de traviesa "JJM con armadura pretesa", sino que en su lugar se ensayó una traviesa monobloque, que fue diseñada con posterioridad al comienzo del Proyecto y está fabricada con armadura postesa.

\section{MEDIOS UTILIZADOS}

Los medios requeridos para el estudio del comportamiento de las traviesas polivalentes son muy diversos, como lo es el conjunto de ensayos diseñados a tal efecto.

En función del tipo de ensayo a realizar, las variables mecánicas a medir, así como las variables de control en cada caso, serían:

- Estáticos: Carga y deformación (control sobre la carga).

- Fatiga: Carga (control sobre la carga), frecuencia y número de ciclos.

- Dinámicos: Carga, deformación, aceleración y desplazamiento (control sobre la carga y la frecuencia).

- Via: Deformación y aceleración (control sobre el eje del coche a medir).

Para cubrir estos aspectos se utilizaron los siguientes equipos:

\subsection{Equipos de carga}

Se emplean dos equipos distintos: uno para los ensayos estáticos y otro para los dinámicos y de fatiga. $\mathrm{Pa}$ ra los ensayos estáticos se utilizó una prensa con una fuente hidráulica capaz de producir una presión de 300 atm., que con los cilindros hidráulicos utilizados supone una carga posible sobre la traviesa de 60 toneladas por punto de carga. Esta prensa incluye un arma- rio de control mediante el cual se pueden producir rampas de carga con velocidad controlada tanto en tramos ascendentes como descendentes, siguiendo un control de tipo proporcional. Para los ensayos dinámicos se utilizó una fuente hidráulica que trabaja con cilindros capaces de proporcionar hasta $\pm 64 \mathrm{t}$ de carga dinámica con la posibilidad de seguir distintas formas de onda, pudiéndose variar la amplitud y la frecuencia de éstas.

Si bien el sistema electrónico analógico permite controlar el ensayo sobre las variables carga o desplazamiento, en este caso el control se realizó a través de la carga.

\subsection{Equipos de medida}

En los ensayos estáticos se midieron las variables carga y deformación. La carga se midió en $\mathrm{kp}$ desde el armario de control de la prensa estática y la deformación en $\mu \mathrm{D}$ mediante un equipo que incluye una caja de conmutación y un voltímetro con ajuste de cero y de fondo de escala, al cual se conectaron las bandas extensométricas.

En los ensayos de fatiga se empleó el equipo incluido en el armario de control de la prensa dinámica que permitía medir cargas, desplazamientos, frecuencia y número de ciclos. La variable de control fue la carga, que se midió en $\mathrm{kp}$.

Para los ensayos dinámicos se utilizó la misma instrumentación que en el caso anterior, pero añadiendo un sistema de adquisición de datos en tiempo real con capacidad para efectuar un muestreo de un millón de muestras por segundo nominalmente. Este equipo incluye un conjunto de programas para configurar la adquisición, tratamiento y almacenamiento de los datos. Se incluyen en él tarjetas de interfase analógicodigitales para poder muestrear hasta 32 canales. A estos canales van a parar las señales correspondientes a las variables de desplazamiento, carga, deformación y aceleración.

En las auscultaciones en vía, la instrumentación empleada coincide con la de los ensayos dinámicos, salvo que la carga no es producida por un equipo hidráulico, sino que es variable ya que la produce el propio equipo móvil.

\subsection{Transductores}

Los diferentes tipos de transductores empleados en los procesos de medida fueron:

- Deformación: Bandas extensométricas autocom- 
pensadas en temperatura, con un diseño geométrico adecuado para detectar deformaciones uniaxiles, longitud de $30 \mathrm{~mm}$ y con disposición en $1 / 4 \mathrm{de}$ puente.

- Desplazamiento: Captadores de desplazamiento tipo LVDT con ajuste de cero y de fondo de escala.

- Aceleración: Se eligieron unos acelerómetros piezoresistivos con banda pasante hasta $100 \mathrm{~Hz}$ que aseguran una sensibilidad a partir de $0 \mathrm{~Hz}$, lo que no ocurre con los piezo-eléctricos ni con los inductivos.

- Carga: Se han utilizado para medir la carga en todos los casos sensores de presión. En el caso de los ensayos estáticos se utilizaron sensores de presión directa y en el resto sensores de presión diferencial, lo que implica medir la carga en el circuito de la presión.

El sistema amplificador elegido en todos los casos es del tipo de frecuencia portadora con posibilidad de ajuste de la línea de base y del fondo de escala. Las ventajas que proporciona este tipo de amplificador son: la estabilidad del cero (no hay deriva del cero) y la estabilidad de la ganancia. No tienen la anchura de banda que proporciona un amplificador de continua, pero se escogió una frecuencia portadora que permitía alcanzar el ancho de banda de medida, que es de $100 \mathrm{~Hz}$.

\section{REALIZACION DE LAS PRUEBAS}

Si bien se han realizado todas las pruebas contempladas en el Plan de Ensayos, hasta la fecha no se ha podido establecer una correlación entre los resultados experimentales, dado que las características de fabricación de las traviesas no han sido uniformes y aún se está elaborando la información correspondiente.

Estas características no uniformes han sido:

- Resistencias obtenidas en las probetas de los hormigones con los que se confeccionaron cada una de las distintas traviesas.

- Posición y características mecánicas de las armaduras colocadas en cada caso.

- Tensión de tesado alcanzada en la armadura de cada una de las traviesas.

Para el planteamiento de los ensayos estáticos y de fatiga, se tuvieron en cuenta en primer lugar los momentos a soportar por la traviesa, y que en función de las hipótesis de cálculo realizadas previamente fueron:

\begin{tabular}{|l|c|c|c|c|}
\hline \multirow{2}{*}{ HIPÓTESIS } & \multicolumn{2}{|c|}{ MBC } & \multicolumn{2}{c|}{ MCV } \\
\cline { 2 - 5 } & A. RENFE & A. INTER. & A. RENFE & A. INTER. \\
\hline $\begin{array}{l}\text { Sin apoyo en la } \\
\text { zona central }\end{array}$ & 0,77 & 1,65 & 0,20 & 0,51 \\
\hline $\begin{array}{l}\text { Apoyo en toda } \\
\text { la traviesa }\end{array}$ & 0,51 & 1,45 & $-1,39$ & $-0,43$ \\
\hline
\end{tabular}

y en segundo lugar los momentos últimos de la traviesa que, para el caso de traviesa con armadura postesa, son:

\begin{tabular}{|c|c|c|c|}
\hline \multicolumn{2}{|c|}{ MBC } & \multicolumn{2}{c|}{ MCV } \\
\hline A. RENFE & A. INTER. & POSITIVO & NEGATIVO \\
\hline 3,27 & 3,08 & 2,30 & $-2,62$ \\
\hline
\end{tabular}

Con estos valores, teniendo en cuenta que el momento positivo máximo en el centro de la traviesa es 0,51 $\mathrm{m} \cdot t$ y el momento último $2,3 \mathrm{~m} \cdot \mathrm{t}$, lo que da un cociente mayor de 4,50, y que la hipótesis de cálculo en que se producen momentos positivos en el centro de la traviesa se da únicamente después de batear y por periodos de tiempo poco prolongados, se decidió centrar los ensayos en el momento positivo bajo carril y en el momento negativo en el centro del vano.

Los ensayos estáticos realizados tenían como misión obtener los momentos flectores que provocan determinados estados de fisuración en la traviesa, así como el momento flector último alcanzado en las distintas hipótesis de comportamiento de las secciones del elemento estructural.

En los ensayos se determinaron los valores de los siguientes momentos flectores:

- Momento de inicio de la fisura $\left(M_{F}\right)$. Es el que produce la primera fisura continua de longitud mayor de $15 \mathrm{~mm}$.

- Momento de fisura de $0,10 \mathrm{~mm}\left(\mathrm{M}_{0,10}\right)$. Es el que produce una abertura de la fisura de $0,10 \mathrm{~mm}$ de an cho.

- Momento de fisura de 0,20 mm $\left(M_{0,20}\right)$. Es el que produce una abertura de la fisura de $0,20 \mathrm{~mm}$ de ancho.

- Momento de rotura $\left(M_{R}\right)$.

La disposición de los ensayos se decidió a partir de los planteamientos anteriores y teniendo en cuenta las características específicas de las traviesas objeto del estudio. 
El primer ensayo se realizó situando la traviesa en posición invertida respecto a su colocación en la vía. En la cara inferior apoyaba sobre dos rótulas separadas $1,50 \mathrm{~m}$ y equidistantes del eje de la traviesa, con una placa de contacto entre la rótula y la traviesa de $14 \mathrm{~cm}$ de ancho, que equivalía al ancho del carril. La carga se aplicó a través de una placa metálica en el centro de la cara superior de la traviesa por medio de un gato hidráulico (Fig. 1).

Las cargas se aplicaron de forma escalonada. En el primer escalón se alcanzaron los $3.200 \mathrm{kp}$ de carga, la cual produce un momento flector de $1,2 \mathrm{~m} \cdot \mathrm{t}$, que es del mismo orden que el máximo que se produce en el centro de la traviesa (1,39 m·t) bajo las hipótesis de cálculo más desfavorables, y para el cual no debiera producirse ninguna fisura. En los sucesivos escalones se aplicaron incrementos de carga de $200 \mathrm{kp}$, equivalentes a un momento flector de $0,075 \mathrm{~m} \cdot \mathrm{t}$. La velocidad de aplicación de las cargas fue de $1.000 \mathrm{kp} / \mathrm{min}$ y el tiempo entre escalones de 5 minutos.

La rotura se produjo en la mayoría de los casos por flexión en la sección central de la traviesa, excepto algún caso en que ésta se produjo por cortante (foto 1) o por fallo de los anclajes (foto 2).

Para verificar la aparición de las fisuras y para medir su ancho se utilizó un microscopio de 25 aumentos, además de situar en la cara inferior de la traviesa, en la zona donde era previsible la aparición de la fisura, una banda extensométrica cuya lectura después de cada escalón de carga facilitó su detección (foto 3).

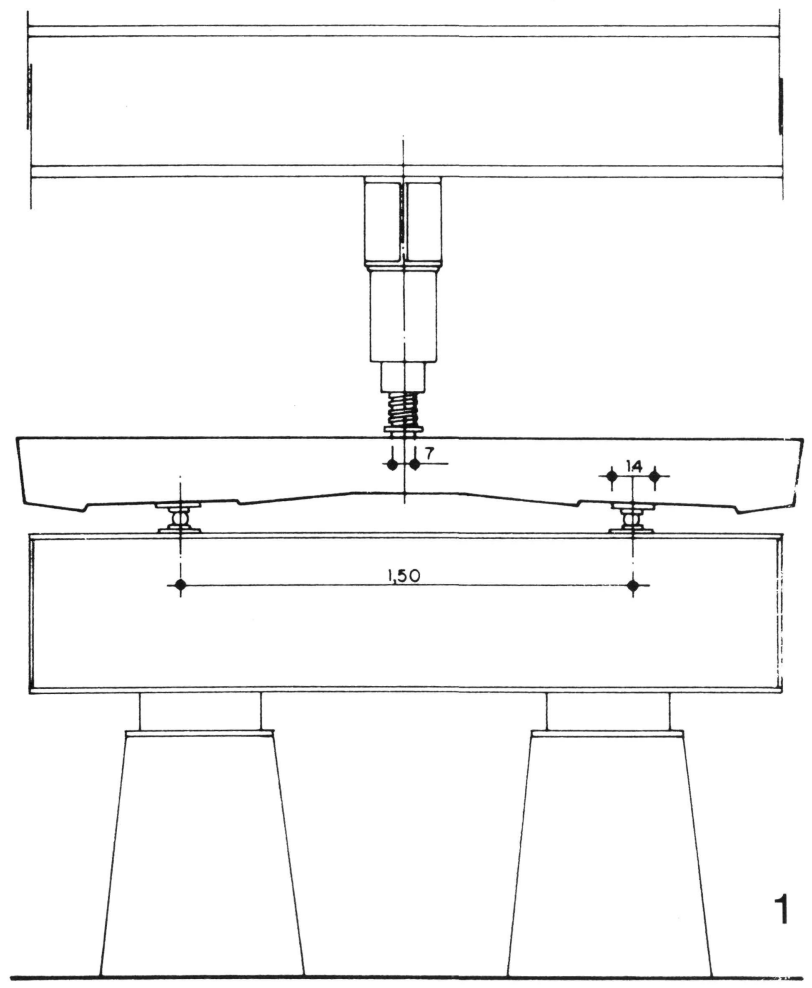

Ensayo estático. Momento negativo en sección central de la traviesa. Carga en el centro del vano.

(c) Consejo Superior de Investigaciones Científicas Licencia Creative Commons 3.0 España (by-nc)

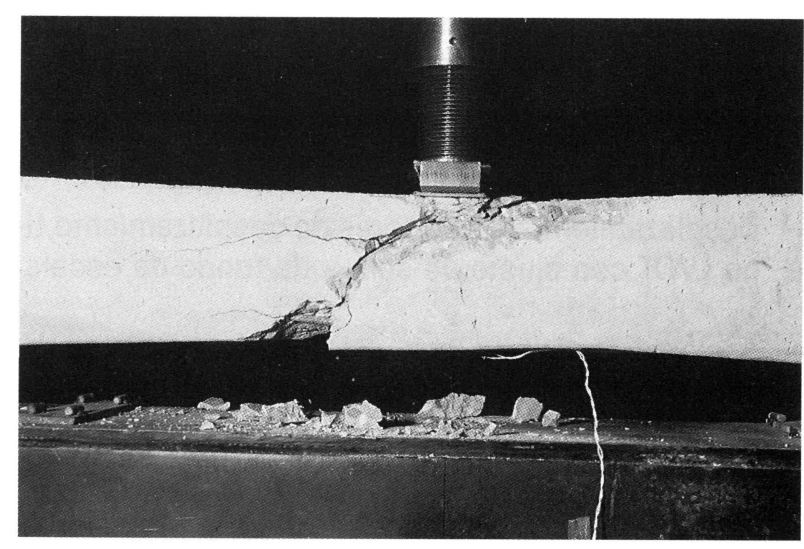

Foto 1

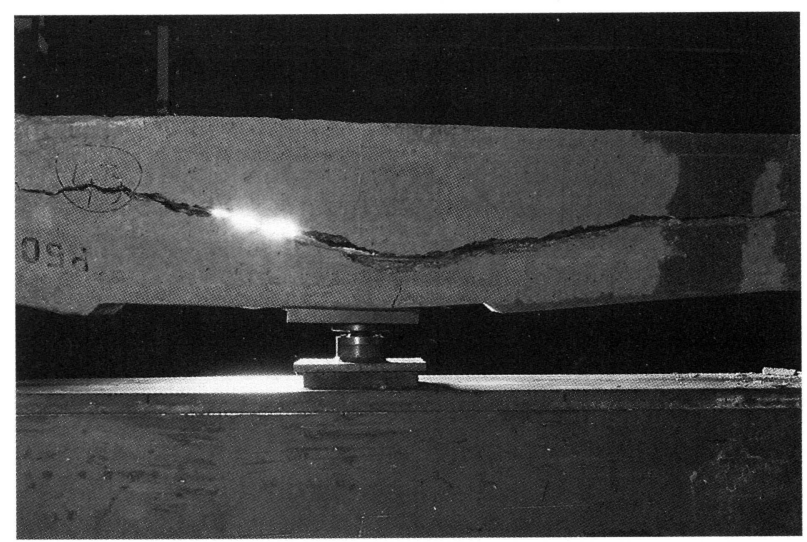

Foto 2

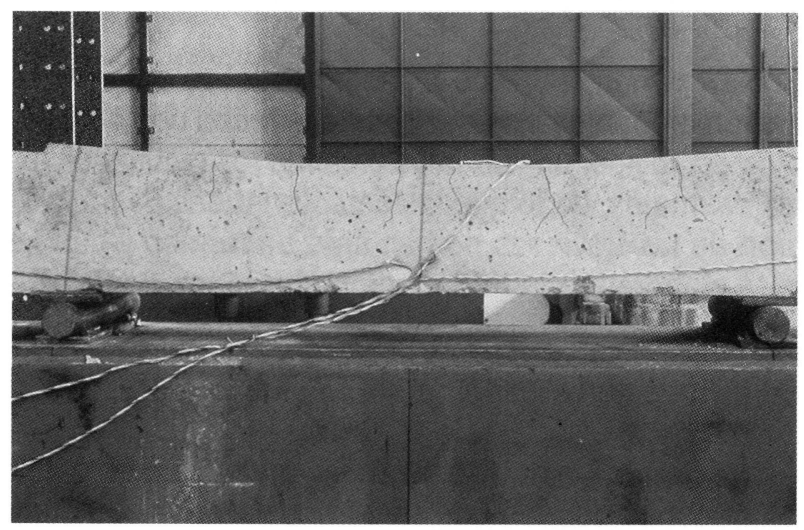

Foto 3

El segundo ensayo se realizó situando la traviesa con su cara inferior apoyada en cuatro puntos. Dado que en este caso la sección con el momento bajo carril más desfavorable es diferente según se trate de ancho RENFE o INTERNACIONAL, el eje de la carga se situó a $0,422 \mathrm{~m}$ en el primer caso y a 0,538 $\mathrm{m}$ en el segundo. Los puntos de apoyo se situaron simétricamente respecto al eje de la traviesa y a una distancia de éste de 40 y $105 \mathrm{~cm}$ respectivamente. Los apoyos consistian http://informesdelaconstruccion.revistas.csic.es 


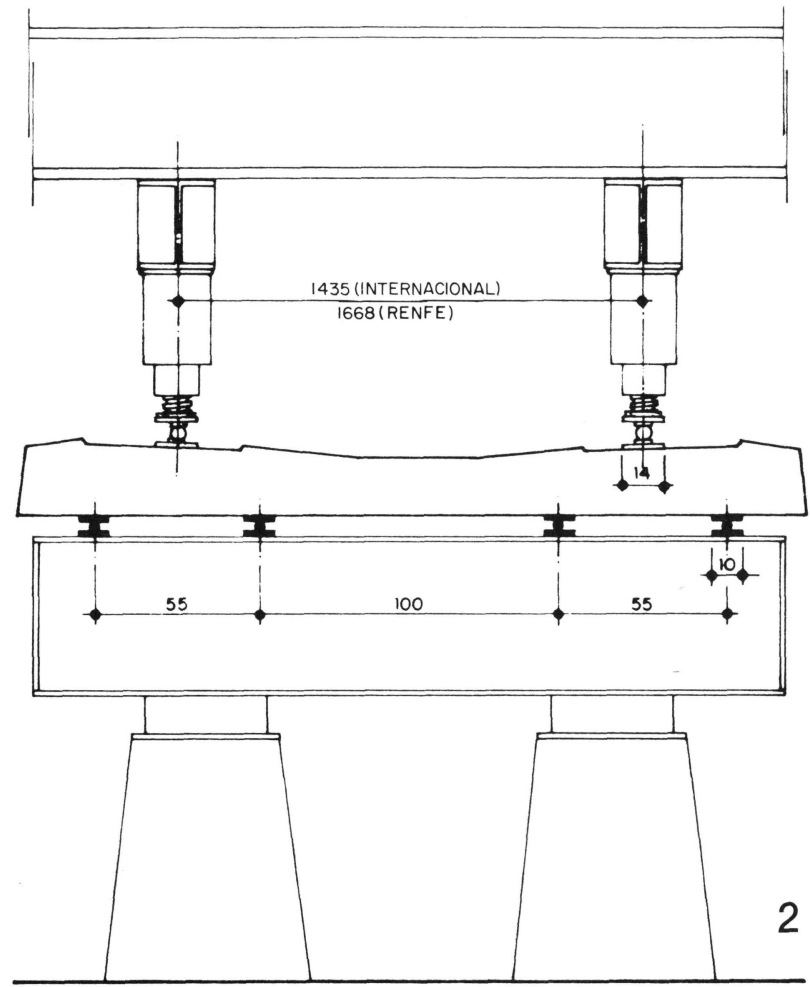

Ensayo estático. Momento bajo carril.

en rodillos metálicos situados entre dos placas de $10 \mathrm{~m}$ de ancho. La carga se aplicó en dos puntos situados simétricamente respecto al eje de la traviesa y con una separación entre ellos de $1.668 \mathrm{~mm}$ (posición ancho RENFE) o $1.435 \mathrm{~mm}$ (posición ancho INTERNACIONAL), mediante dos gatos hidráulicos, a través de dos rótulas que apoyaban en placas de $14 \mathrm{~cm}$ de ancho, equivalente al del carril (Fig. 2).

Como en el ensayo anterior las cargas se aplicaron de manera escalonada. En el primer escalón se alcanzaron los $17.500 \mathrm{kp}$ por gato, lo que equivalia a un momento flector de 1,86 $\mathrm{m} \cdot \mathrm{t}$ en ancho INTERNACIONAL y a $2 \mathrm{~m}$ 't en ancho RENFE, superiores ambos a los momentos bajo carril que aparecen en la traviesa en cualquiera de los dos anchos. En los escalones sucesivos se aplicaron incrementos de carga de $1.000 \mathrm{kp}$ (equivalente a un momento flector de 0,106 y $0,113 \mathrm{~m} \cdot \mathrm{t}$ respectivamente). La velocidad de aplicación de las cargas fue de $1.000 \mathrm{kp} / \mathrm{min}$ y el tiempo entre escalones de $5 \mathrm{mi}$ nutos. Como con esta disposición del ensayo se producían momentos negativos en el centro de la traviesa, se colocaron 3 bandas extensométricas, una en el centro de la cara superior de la traviesa y otras dos en la cara inferior bajo los gatos, a las que se tomó lectura en cada escalón de carga y que ayudaron a detectar las fisuras.

La rotura se produjo en todos los casos por cortante, variando la sección en la que ésta se producía según el ensayo fuera con ancho RENFE (foto 4) o INTERNACIONAL (foto 5).

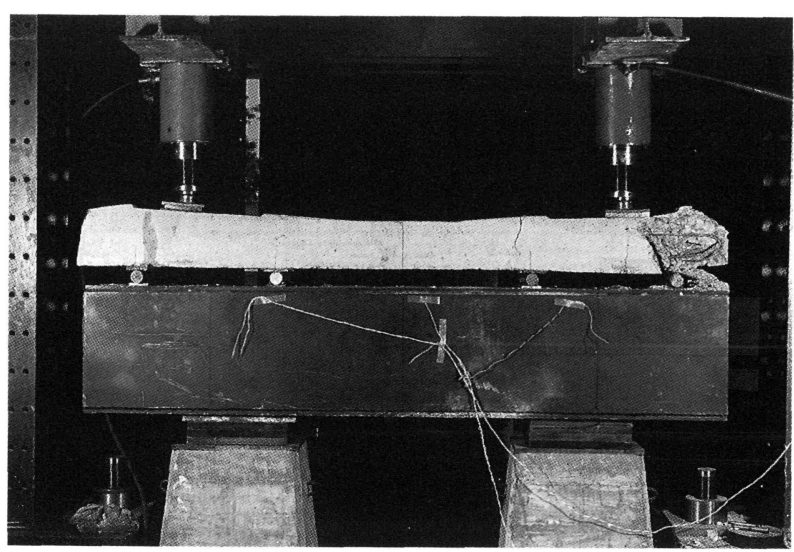

Foto 4

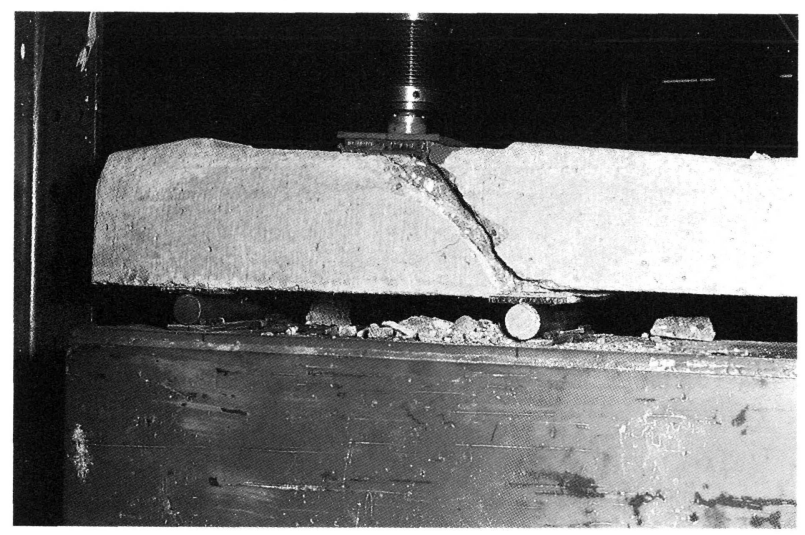

Foto 5

Los ensayos de fatiga se plantearon con objeto de observar el estado de fisuración de la traviesa después haber recibido un determinado número de ciclos de carga, para a continuación ser sometida al ensayo estático correspondiente y comprobar la posible variación en los resultados obtenidos respecto de los de las traviesas sin fatigar.

Según la línea de Wöhler, la resistencia a tracción del hormigón decrece con el número de ciclos hasta estabilizarse una vez que ha recibido del orden de dos miIlones de ciclos. Teniendo en cuenta el número de traviesas a ensayar y las frecuencias máximas que se podían alcanzar en el ensayo, para que la duración de éste no fuera excesiva se pensó ensayar las traviesas en cuatro grupos, en cada uno de los cuales el número de ciclos sería de $100.000,500.000,1.000 .000$ y 2.000 .000 . En cuanto a la frecuencia, fue ésta de $15 \mathrm{~Hz}$ habiéndose comprobado previamente instalando captadores de desplazamiento en determinados puntos de la traviesa, que los desplazamientos estaban en fase con las cargas y que no aumentaba su amplitud con la frecuencia.

El primer ensayo a fatiga se realizó situando la traviesa en posición invertida respecto a su colocación en 


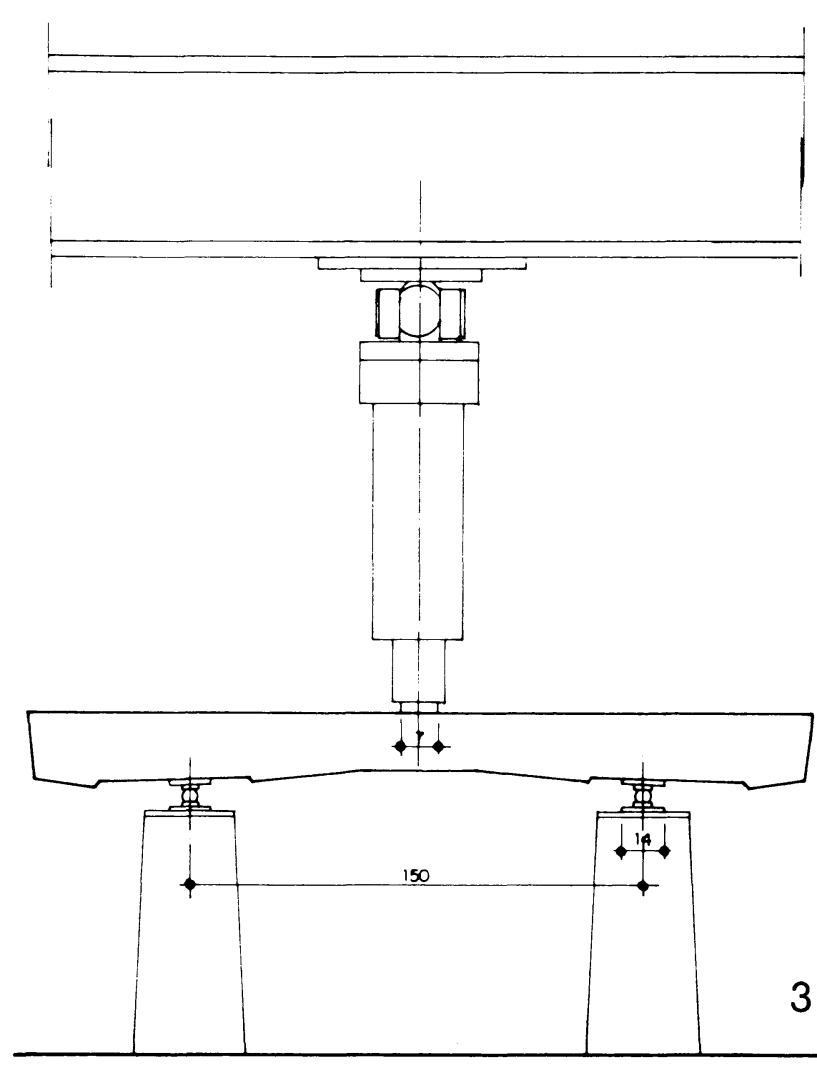

Ensayo de fatiga. Momento negativo en sección central de la traviesa.

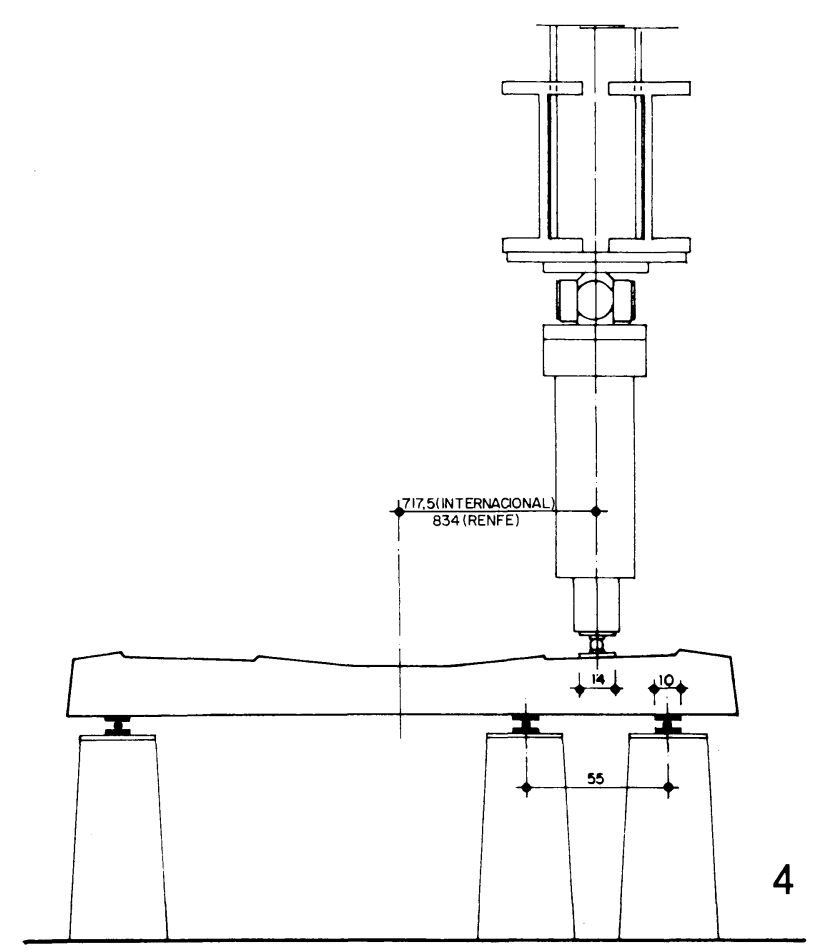

Ensayo de fatiga. Momento bajo carril. la vía, con la misma disposición que el primer ensayo estático que determinaba el momento flector negativo en la sección central (Fig. 3).

Las cargas se aplicaron siguiendo una onda sinusoidal oscilando entre 1.000 y $5.000 \mathrm{kp}$, lo que produce unos momentos en el centro del vano comprendidos entre 0,375 y $1.875 \mathrm{~m} \cdot \mathrm{t}$, momentos que son del orden del $15 \%$ y del $75 \%$ del momento último y el máximo, superior al momento de servicio de la traviesa. Al terminar el número de ciclos correspondiente a cada traviesa y tras observar su estado de fisuración, tanto en carga como descargada, se la sometía al ensayo estático correspondiente.

El segundo ensayo se realizó aplicando la carga sólo sobre una de las secciones bajo carril. Por tanto la traviesa se colocó apoyando su cara inferior en dos puntos situados a 50 y $105 \mathrm{~cm}$ del eje de la traviesa respectivamente. Dichos apoyos consistían en rodillos metálicos situados entre dos placas de $10 \mathrm{~cm}$ de anchura (Fig. 4).

3 Como se ha mencionado anteriormente, al cambiar la posición del carril sobre la traviesa cambia la sección sometida a mayor momento. Al haberse obtenido en el cálculo los momentos últimos correspondientes a la sección bajo carril en las dos hipótesis de ancho, se pensó realizar el ensayo para comprobar las dos secciones, sometiendo un grupo de traviesas al ensayo de fatiga correspondiente a ancho RENFE para pasarlas después al ensayo estático correspondiente a este ancho, y otro grupo ensayarlas a fatiga en ancho internacional para someterlas después al ensayo estático con este mismo ancho.

Así se hizo cuando se empezaron los ensayos con la traviesa JJM, a pesar de que en esta traviesa las cargas del carril se aplicaban a través de una placa metálica de $495 \times 165 \times 25 \mathrm{~mm}$, cuyo efecto era el de repartir las cargas del carril en toda esta superficie, por lo que el cambio de posición del carril no producía un efecto $\tan$ apreciable.

Pero durante la realización de las primeras pruebas, se cambió el plan de ensayos al decidir RENFE sustituir los correspondienes a la traviesa JJM con armadura pretesa por otros sobre una nueva traviesa. Ésta consistía en una traviesa monobloque de hormigón armado con armadura postesa, con un sistema de sujeción constituido por una grapa metálica que apoyaba en la base del carril y en dos placas metálicas, de perfil geométrico diferente, alojadas en sus extremos en un rebaje en la base de la traviesa. Las placas se fijaban a la traviesa por medio de pernos que roscaban en unos alojamientos de los que existían cuatro en cada extremo de ella. El cambio de ancho se conseguía al utilizar una u otra pareja de alojamientos para los pernos. 


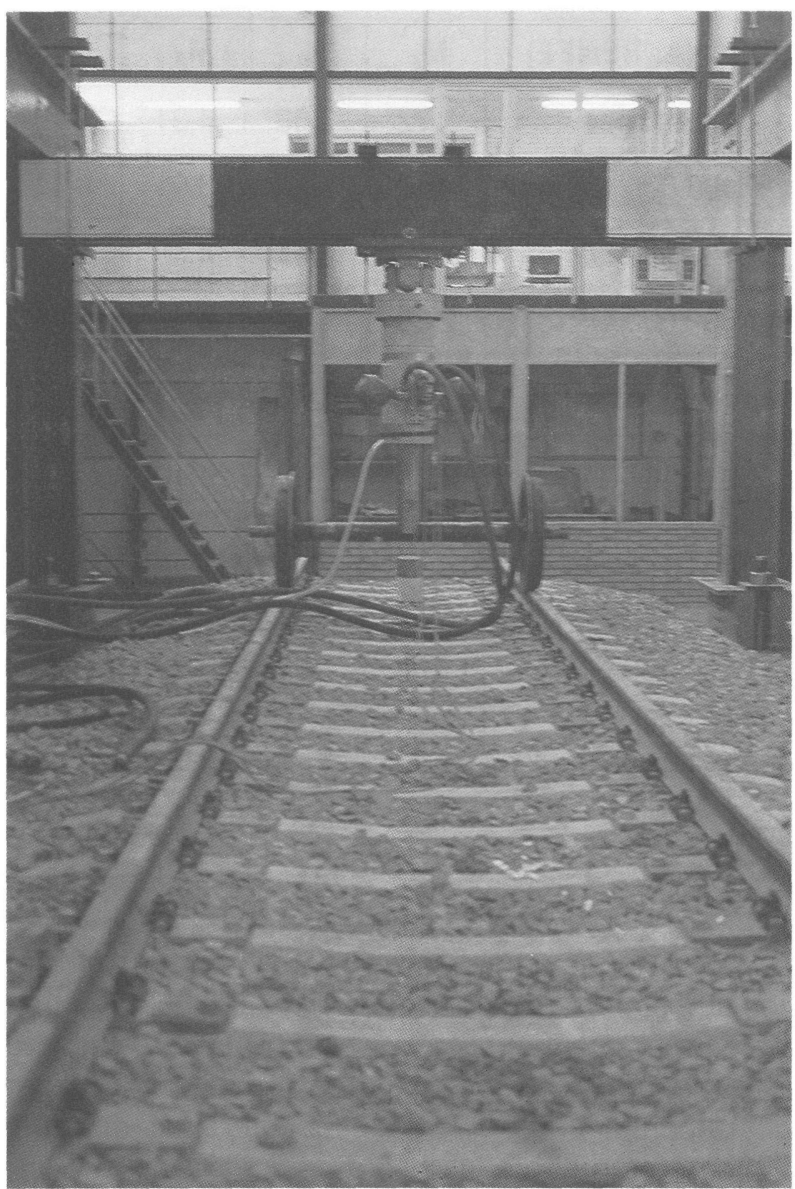

Foto 6

Como en esta traviesa el carril apoyaba directamente sobre ella, se planteaba con más necesidad el estudio de las dos secciones bajo carril, por lo que se decidió someter a todas las traviesas al número correspondiente de ciclos en la posición de ancho RENFE y al mismo número en la posición de ancho internacional, para efectuar después sobre ellas el ensayo estático correspondiente.

La carga se aplicó en un punto situado a 834 mm ó 715,5 $\mathrm{mm}$, según el caso, del eje de la traviesa por medio de un actuador hidráulico dinámico a través de una rótula que apoyaba en una placa de $14 \mathrm{~cm}$, equivalente al ancho del carril. Las cargas se introdujeron según una onda sinusoidal oscilando entre 4.500 y $20.000 \mathrm{kp}$, con una frecuencia de $15 \mathrm{~Hz}$. Estas cargas producían en las secciones en estudio momentos comprendidos entre el $12 \%$ y el $85 \%$ de los momentos últimos de diseño de la traviesa y, en ambos casos, superiores a los momentos de servicio.

Para la aplicación de los esfuerzos en los ensayos dinámicos en el tramo de vía de $10 \mathrm{~m}$, construido en el laboratorio, se utilizó un cilindro hidráulico de 64 t, suspendido sobre la vía y transmitiendo las cargas a través de un eje de ferrocarril de los utilizados normalmente como material móvil de RENFE (foto 6).
Las cargas se aplicaron en el punto medio de dicho eje en tres disposiciones diferentes:

- Cilindro en posición vertical, con cargas de 22,5 t.

- Cilindro inclinado $11,3^{\circ}$ respecto a la vertical, en el plano perpendicular a la vía, simulando esfuerzos laterales de valor 4,5 t, para una carga vertical de $22,5 \mathrm{t}$.

- Cilindro inclinado $12,5^{\circ}$ respecto a la vertical, en el plano medio de la vía, simulando esfuerzos longitudinales de $5 \mathrm{t}$, equivalentes a una aceleración máxima de 2,2 m/s², para una carga vertical de 22,5 t.

Los ensayos se realizaron con frecuencias variables, alcanzándose un máximo de $20 \mathrm{~Hz}$, aplicando las cargas por medio de una onda sinusoidal que oscilaba entre $4,5 \mathrm{t}$ y $22,5 \mathrm{t}$.

Con objeto de simular posibles defectos del material, se hicieron otros ensayos en los que a la onda anterior se superpuso otra, de manera que cada 100 ciclos se aplicaba una carga de $40 \mathrm{t}$.

Se instrumentaron las traviesas con bandas extensométricas, acelerómetros y captadores de desplazamiento, midiéndose los datos correspondientes a la traviesa situada bajo el eje y las dos adyacentes a ambos lados.

Para los ensayos realizados en un tramo de la Red se instrumentaron las traviesas de forma análoga a la descrita para el tramo experimental, obteniéndose los datos al paso de los distintos vehículos.

En cuanto a los resultados obtenidos es de destacar que en los ensayos para la determinación del momento negativo en el centro del vano, existieron variaciones entre las traviesas procedentes de distintos fabricantes. Para tres de ellos no se apreciaron entre las traviesas sometidas a fatiga y sin fatigar otras diferencias que las propias de los elementos de hormigón, tal como puede verse en la tabla siguiente, obtenida con la media de los valores correspondientes a las traviesas ensayadas de estos tres fabricantes.

\begin{tabular}{|c|c|c|c|c|}
\hline MCV & $M_{F}$ & $M_{0,1}$ & $M_{0,2}$ & $M_{R}$ \\
\hline Estático & 2,27 & 2,83 & 3,02 & 4,56 \\
\hline 100.000 Ciclos & 2,52 & 3,12 & 3,28 & 4,48 \\
\hline 500.000 Ciclos & 2,20 & 3,00 & 3,15 & 4,59 \\
\hline 1.000.000 Ciclos & 2,54 & 3,07 & 3,19 & 4,57 \\
\hline 2.000 .000 Ciclos & 2,90 & 3,16 & 3,20 & 4,48 \\
\hline
\end{tabular}


En las traviesas del cuarto fabricante si se detectaron diferencias entre las sometidas a los ensayos de fatiga y las no fatigadas, según se aprecia en la tabla siguiente, obtenida con las medias de los valores correspondientes a las traviesas de este último fabricante.

\begin{tabular}{|c|c|c|c|c|}
\hline $\mathbf{M C V}$ & $\mathbf{M}_{\mathbf{F}}$ & $\mathbf{M}_{\mathbf{0 , 1}}$ & $\mathbf{M}_{\mathbf{0 , 2}}$ & $\mathbf{M}_{\mathbf{R}}$ \\
\hline Estático & 2,26 & 2,64 & 2,72 & 4,07 \\
\hline 100.000 Ciclos & 2,37 & 2,90 & 3,09 & 4,30 \\
\hline 1.000 .000 Ciclos & 1,58 & 2,27 & 2,56 & 4,22 \\
\hline 2.000 .000 Ciclos & 1,81 & 2,18 & 2,56 & 3,69 \\
\hline
\end{tabular}

Con relación a los ensayos para la determinación del momento bajo carril se puede decir, tal como se aprecia en la tabla que figura a continuación, que la fatiga afecta ligeramente a los valores de los momentos de fisuración pero no asi al momento de rotura.

\begin{tabular}{|l|c|c|c|c|}
\hline $\mathbf{M B C}$ (A. RENFE) & $\mathbf{M}_{\mathbf{F}}$ & $\mathbf{M}_{\mathbf{0 , 1}}$ & $\mathbf{M}_{\mathbf{0 , 2}}$ & $\mathbf{M}_{\mathbf{R}}$ \\
\hline Estático & 3,25 & 3,63 & 3,86 & $>6,04$ \\
\hline 100.000 Ciclos & 2,72 & 3,06 & 3,28 & $>6,15$ \\
\hline 500.000 Ciclos & 2,54 & 3,00 & 3,17 & $>6,09$ \\
\hline 1.000 .000 Ciclos & 2,77 & 2,94 & 3,05 & $>6,14$ \\
\hline
\end{tabular}

Por último, los ensayos realizados sobre el segundo modelo de traviesa quedan recogidos en las tablas siguientes en las que se reflejan los valores medios de los resultados obtenidos, y de los que únicamente puede deducirse un ligero descenso del valor del momento de comienzo de la fisuración con la fatiga, en el ensayo para la determinación del momento en el centro del vano. En el resto de los casos los esfuerzos de fatiga no afectan al comportamiento de la traviesa.

\begin{tabular}{|c|c|c|c|c|}
\hline $\mathbf{M C V}$ & $\mathbf{M}_{\mathbf{F}}$ & $\mathbf{M}_{\mathbf{0 , 1}}$ & $\mathbf{M}_{\mathbf{0 , 2}}$ & $\mathbf{M}_{\mathbf{R}}$ \\
\hline Estático & 2,90 & 3,20 & 3,35 & 4,56 \\
\hline 100.000 Ciclos & 2,75 & 3,31 & 3,47 & 4,56 \\
\hline 500.000 Ciclos & 2,52 & 3,24 & 3,39 & 4,63 \\
\hline 1.000 .000 Ciclos & 2,11 & 2,60 & 3,01 & 4,56 \\
\hline
\end{tabular}

(c) Consejo Superior de Investigaciones Científicas Licencia Creative Commons 3.0 España (by-nc)

\begin{tabular}{|c|c|c|c|c|}
\hline MBC (A. RENFE) & $\mathbf{M}_{\mathbf{F}}$ & $\mathbf{M}_{\mathbf{0 , 1}}$ & $\mathbf{M}_{\mathbf{0 , 2}}$ & $\mathbf{M}_{\mathbf{R}}$ \\
\hline Estático & 2,72 & 3,68 & 4,31 & 6,12 \\
\hline 100.000 Ciclos & 2,43 & 3,68 & 3,91 & 6,11 \\
\hline 500.000 Ciclos & 2,54 & 3,91 & 4,36 & 6,13 \\
\hline 1.000 .000 Ciclos & 2,60 & 4,08 & 4,36 & 6,11 \\
\hline
\end{tabular}

\begin{tabular}{|c|c|c|c|c|}
\hline MBC (A. INT.) & $\mathbf{M}_{\mathbf{F}}$ & $\mathbf{M}_{\mathbf{0 , 1}}$ & $\mathbf{M}_{\mathbf{0 , 2}}$ & $\mathbf{M}_{\mathbf{R}}$ \\
\hline Estático & 2,71 & 3,72 & 4,04 & 5,24 \\
\hline 100.000 Ciclos & 2,71 & 3,89 & 4,10 & 5,64 \\
\hline 500.000 Ciclos & 2,39 & 4,42 & 4,53 & 5,66 \\
\hline 1.000 .000 Ciclos & 2,60 & 3,29 & 3,88 & 5,69 \\
\hline
\end{tabular}

La primera explicación que se obtiene de estos resultados es que las cargas que se adoptaron para los ensayos de fatiga, si bien eran acordes con los esfuerzos que soportarian en la vía, estaban muy por debajo de los valores últimos capaces de soportar por las traviesas $y$, por tanto, las tensiones producidas estaban por debajo del umbral en que los esfuerzos de fatiga tienen efecto sobre las traviesas.

Esto se pone de manifiesto claramente al comprobar que los valores de los momentos últimos de rotura apenas sufren variación al someter a las traviesas a fatiga, mientras que esta variación, aunque muy leve, es apreciable, en la mayoría de las pruebas realizadas, para el valor del momento de inicio de la fisuración.

Por otro lado, en todos los casos, los valores de los momentos de inicio de la fisuración resultaron ser superiores a los momentos que, previsiblemente, soportará la traviesa en servicio en la vía, por lo que el diseño de las traviesas polivalentes se consideró como satisfactorio para responder a las acciones que el tráfico previsto en la Red fuera a introducir.

\section{CONCLUSIONES}

La primera conclusión que se obtiene de los estudios ya terminados y de los que están en curso es el buen comportamiento de las traviesas y la gran correlación que se ha obtenido con las previsiones teóricas formuladas.

Es de destacar que, si bien la traviesa JJM ha mostrado un mejor comportamiento, los resultados de la del otro modelo ensayado han sido también satisfactorios, por lo que la posibilidad de utilizarlas en la Red ferroviaria se ha determinado como técnicamente factible. 
Hay que señalar aquí que el coste de la traviesa JJM es superior al de los otros modelos aparecidos, al incorporar una placa metálica que, si bien reparte mejor las cargas a la traviesa y permite incorporar una sujeción indirecta, cuya eficacia parece mayor, tiene un precio relativamente importante respecto del coste total del elemento estructural.

Es de destacar el hecho de que este procedimiento de cambio de ancho, que se está desarrollando en España con tecnología y diseños totalmente españoles, ha despertado gran interés en la Comunidad Ferroviaria Internacional, que sigue de cerca los avances que se van produciendo, manteniendo un contacto continuo con los técnicos e investigadores que desarrollan el Proyecto.

A la vista de los resultados de los estudios técnicos y económicos, parece que se ha podido llegar a encontrar una solución que respondiera a todas las cuestiones planteadas para resolver técnicamente el cambio de ancho de vía en España.

Esta solución podría ser la sustitución progresiva de las traviesas existentes en la Red, por otras monobloque polivalentes, aprovechando que una sustitución de traviesas se debe ir produciendo por la propia operativa de mantenimiento de la infraestructura ferroviaria. Esta incorporación de traviesas polivalentes no supondría, en sí misma, un incremento de inversión significativo, ya que el coste de estas traviesas y las monobloque actuales es muy parecido. Se llegaría así a que dentro de 20 años, aproximadamente, podrían estar las principales arterias de la Red con una infraestructura que permitiera, desde un punto de vista técnico, cambiar el ancho de vía a 1,435 m.

En nuestra opinión, ése sería el momento más adecuado para que se produjeran las extrapolaciones de los estudios económicos, pues las condiciones de contorno pueden variar a lo largo de estos próximos años, invalidando, o al menos minimizando, las conclusiones socio-económicas de dichos estudios. En cualquier caso, la infraestructura estaría dispuesta para poder cambiar el ancho en el momento que se considerara oportuno, bien en toda la Red, bien en aquellas líneas que pudiera interesar.

Hay otros aspectos que no se han comentado aqui por salirse del objeto de la Investigación realizada pero que tienen una incidencia importantísima en la decisión del cambio de ancho, como son el material móvil, el tendido eléctrico, los cruces y acceso a estaciones,...

Un último comentario que hay que hacer acerca de las investigaciones en infraestructura ferroviaria y de la aplicación de nuevas tecnologías en la construcción de la misma, es la posibilidad de realizar un Proyecto tendente al desarrollo de la vía sustentada en placa de hormigón, para utilizarla en puentes, en túneles, en paso por zonas urbanas, en estaciones, en intersecciones,..., y solucionar alguno de los problemas de ruidos, vibraciones en edificios, replanteo en planta del trazado,... existentes en la actualidad y que por ahora no han podido resolverse en su totalidad. En ese sentido hay que señalar que ya se han iniciado las gestiones para que en un futuro próximo se comiencen a realizar los primeros estudios que lleven a poder diseñar una solución de vía en placa con patente y tecnología española. 